\title{
PAIRS OF ADDITIVE CONGRUENCES TO A LARGE PRIME MODULUS
}

\author{
O. D. ATKINSON AND R. J. COOK
}

(Received 13 October 1987; revised 11 May 1988)

Communicated by J. H. Loxton

\begin{abstract}
This paper is concerned with non-trivial solvability in $p$-adic integers, for relatively large primes $p$, of a pair of additive equations of degree $k>1$ :

$$
\begin{aligned}
& f(\mathbf{x})=a_{1} x_{1}^{k}+\cdots+a_{n} x_{n}^{k}=0, \\
& g(\mathbf{x})=b_{1} x_{1}^{k}+\cdots+b_{n} x_{n}^{k}=0,
\end{aligned}
$$

where the coefficients $a_{1}, \ldots, a_{n}, b_{1}, \ldots, b_{n}$ are rational integers.

Our first theorem shows that the above equations have a non-trivial solution in $p$-adic integers if $n>4 k$ and $p>k^{6}$. The condition on $n$ is best possible.

The later part of the paper obtains further information for the particular case $k=5$. Specifically we show that when $k=5$ the above equations have a non-trivial solution in $p$-adic integers (a) for all $p>3061$ if $n \geq 21$; (b) for all $p$ except $p=5$, 11 if $n \geq 26$.

1980 Mathematics subject classification (Amer. Math. Soc.) (1985 Revision): 11 D 88.
\end{abstract}

\section{Introduction}

It is well known (see, for example, Chapter 1 of Borevich and Shafarevich [3]) that the number of solutions of a polynomial congruence

$$
F\left(x_{1}, \ldots, x_{n}\right) \equiv 0 \quad \bmod p
$$

may be estimated using exponential sums. For an additive form

$$
a_{1} x_{1}^{k}+\cdots+a_{n} x_{n}^{k} \equiv 0 \quad \bmod p,
$$

(C) 1989 Australian Mathematical Society $0263-6115 / 89 \$ A 2.00+0.00$ 
where $p \nmid a_{1} \cdots a_{n}$, it follows from Theorem B of Borevich and Shafarevich [3, page 15] that the number $N$ of solutions of (1) satisfies

$$
\left|N-p^{n-1}\right| \leq C p^{(n / 2)-1},
$$

with $C=(k-1)^{n}$. Therefore a congruence

$$
a x^{k}+b y^{k}+c z^{k} \equiv 0 \quad \bmod p, p \nmid a b c,
$$

has a non-trivial solution for all $p>k^{6}$. The condition on $p$ may be improved to $p>k^{4}$ (see Theorem 1 of Chowla [4] or Lemma 2.4.1 of Dodson [17]).

Before considering pairs of additive equations we recall some of the results on the $p$-adic solvability of a single additive equation

$$
f(\mathbf{x})=a_{1} x_{1}^{k}+\cdots+a_{n} x_{n}^{k}=0,
$$

with coefficients in $\mathbf{Z}$. For quadratic forms $(k=2)$ the equation has a nontrivial solution in $p$-adic integers for every prime $p$ provided that $n \geq 5=$ $2.2+1$. This result is best possible since when $n=4$ and $p \equiv 3 \bmod 4$ the equation

$$
x_{1}^{2}+x_{2}^{2}+p\left(x_{3}^{2}+x_{4}^{2}\right)=0
$$

has no non-trivial solution in $p$-adic integers.

For $k=3$ Lewis [20] showed that (4) has a non-trivial solution in $p$-adic integers for every prime provided that $n \geq 7=2.3+1$. In order to see that the condition $n \geq 7$ is best possible, let $p$ be any prime with $p \equiv 1 \bmod 3$ and let $q$ be a cubic non-residue $\bmod p$. Then the equation

$$
\left(x_{1}^{3}-q y_{1}^{3}\right)+p\left(x_{2}^{3}-q y_{2}^{3}\right)+p^{2}\left(x_{3}^{3}-q y_{3}^{3}\right)=0
$$

has no non-trivial solution in $p$-adic integers.

For $k=5$, Gray [19] showed that (4) has a solution in every $p$-adic field provided that $n \geq 16=3.5+1$. This is best possible since the equation

$$
\sum_{i=1}^{5} 11^{i-1}\left(x_{i}^{5}+2 y_{i}^{5}+4 z_{i}^{5}\right)=0
$$

has no non-trivial solution in 11 -adic integers.

Davenport and Lewis [11] showed that for any $k>1$ the equation (4) has a non-trivial solution in $p$-adic integers provided that $n \geq k^{2}+1$. This is best possible for any exponent $k$ such that $k=p-1$ for some prime $p$, as can be seen from a generalization of the example (5); see [11, page 454].

The next theorem is a "folklore" result, which does not seem to appear explicitly in the literature. It follows on combining the arguments of Davenport and Lewis [11] with the result for congruence (3), and the proof is left to the reader. 
TheOREM A. Let $n \geq 2 k+1$. A single additive equation (4) has a non-trivial solution in $p$-adic integers for all $p>k^{4}$.

A generalization of the example (6) shows that the condition $n \geq 2 k+1$ is best possible. The interest of the result is that the problem of $p$-adic solvability is reduced to a finite, and explicit, question; for a given equation the remaining primes can be dealt with by a computer.

Our aim here is to produce an analogue of Theorem A for pairs of additive equations and to exploit this further in the case $k=5$. To gain some idea of what may be feasible for given $k$ and large primes $p$ we consider a generalization of the example (6). For any exponent $k$ and any prime $p \equiv 1$ $\bmod k$, let $q$ be a $k$ th power non-residue $\bmod p$. Then the equation

$$
\sum_{i=1}^{k} p^{i-1}\left(x_{i}^{k}-q y_{i}^{k}\right)=0
$$

has no non-trivial solution in $p$-adic integers. We consider (8) together with a "disjoint copy" of (8) (the equation obtained by replacing $x_{i}, y_{i}$ with new variables $x_{i}^{\prime}, y_{i}^{\prime}$ for $\left.i=1, \ldots, k\right)$. This gives a pair of equations in $4 k$ variables which have no non-trivial solution in $p$-adic integers, no matter how large $p$ is. Thus in order to generalize Theorem $A$ to a pair of additive equations we must at least assume that $n \geq 4 k+1$.

For $k=2$, two quadratic equations (not necessarily additive) have a nontrivial solution in $p$-adic integers for all primes $p$ provided that $n \geq 9$ (see Demyanov [16]), and this result is best possible. For $k=3$, Davenport and Lewis [12] showed that two additive equations

$$
\begin{array}{ll}
f(\mathbf{x})=a_{1} x_{1}^{k}+\cdots+a_{n} x_{n}^{k}=0, & a_{i} \in \mathbf{Z}, \\
g(\mathbf{x})=b_{1} x_{1}^{k}+\cdots+b_{n} x_{n}^{k}=0, & b_{i} \in \mathbf{Z},
\end{array}
$$

have a non-trivial solution in $p$-adic integers for every prime $p$ provided that $n \geq 16$. They also gave a counterexample with $n=15$ and $p=7$ showing that this is best possible. More recently, Cook [7] has shown that for all $p \neq 7$ a sufficient condition is $n \geq 13=4.3+1$. In view of the example (8), and the remarks following it, this result is best possible; if we reduce $n$ to 12 there are infinitely many primes $p(p \equiv 1 \bmod 3)$ for which we have counterexamples.

Davenport and Lewis [14] studied the case of two additive equations (9) with an exponent $k>1$, obtaining sufficient conditions for the equations to have a non-trivial solution in $p$-adic integers for every prime $p$. For odd $k$ they showed that $n \geq 2 k^{2}+1$ variables are sufficient, but for even $k$ they were only able to prove that $n \geq 7 k^{3}$ variables would suffice.

THEOREM 1. Let $n>4 k$. Any two additive equations (9) of degree $k$ with integer coefficients $a_{i}, b_{i}$ have a non-trivial solution in p-adic integers for all 
primes $p>k^{6}$. Further this result is best possible in the sense that it fails to hold when $n=4 k$.

The last sentence of Theorem 1 follows from the remarks following the example (8). We also note that Theorem 1 follows from the results of Demyanov [16] when $k=2$ and Cook [7] when $k=3$, so we may suppose that $k>3$. The case $k=5$ has already been investigated in some detail by Cook [8,9] who showed that $n \geq 31$ variables will suffice expect possibly when $p=11$. Moreover, consideration of two disjoint copies of the equation (7), in a total of 30 variables, shows that the best possible condition for such a result covering all primes $p$ would be $n \geq 31$. However, for $p=11$ Cook [9] was only able to show that $n \geq 41$ variables will suffice.

We investigate those primes $p$ for which the condition $n \geq 21=4.5+1$ is sufficient. Theorem 1 deals with those primes $p>5^{6}=15625$. Some primes $p<5^{6}$ may be dealt with by explicitly calculating exponential sums, and appropriate computer investigation deals with other cases. The primes $p$ for which $n \geq 26=5.5+1$ is sufficient were also investigated by similar methods. The results are summarized in the following theorem.

THEOREM 2. In the case $k=5$ the equations (9) have a non-trivial solution in p-adic integers

(a) for all $p>3061$ when $n \geq 21$;

(b) for all $p$ except $p=5,11$ if $n \geq 26$.

When $p=11$ we have already constructed an example in 30 variables having no non-trivial solutions. Computer searches have revealed examples which may be used to construct similar counterexamples in 25 variables for $p=31$ and 41 . These are listed at the end of this paper.

Apart from their intrinsic interest, $p$-adic solutions are an essential preliminary to any application of the Hardy-Littlewood method. In the case $k=3$, Davenport and Lewis [12] showed that two additive cubic equations have a non-trivial simultaneous solution in rational integers provided that $n \geq 18$. Subsequently ' 18 ' was reduced to ' 17 ' by Cook [6] and ' 16' by Vaughan [24]. In view of the counterexample of Davenport and Lewis [12] with $n=15$ and $p=7$ this is the best that could be done without making some 7-adic assumption. More recently, Baker and Brüdern [2] have shown, using the $p$-adic results of Cook [7], that 15 variables are sufficient if we assume the existence of non-singular 7-adic solutions. Atkinson [1] has classified those pairs of additive cubic equations in $n=13,14$ or 15 variables which do not have 7 -adic solutions. 
The Hardy-Littlewood method requires the existence of non-singular (not just non-trivial) $p$-adic solutions. In view of the recent advances in this method, see for example Vaughan [25], we state (without proof) an appropriate version of Theorem 1 . The point here being that this reduces any $p$-adic assumptions to a finite (and explicit) set of primes.

THEOREM 3. Let $p>k^{4}$ and suppose that the equations (10) have a nontrivial p-adic solution. If every form $\lambda f+\mu g,(\lambda, \mu \neq 0,0)$ in the pencil of $f$ and $g$ has at least $2 k+1$ variables with non-zero coefficients then the equations have a non-singular p-adic solution.

The proof mimics the proofs of Theorem 2 of Davenport and Lewis [14] except that we appeal to Theorem $A$ instead of their result [11] on additive forms in $k^{2}+1$ variables.

One question which naturally arises is how these results generalize to $R>2$ simultaneous equations. An example given by Davenport and Lewis [13, Section 4] shows that the generalization is not straightforward. The $p$-adic results obtained by Davenport and Lewis [15] for $R$ simultaneous equations required $\left[9 R^{2} k \log 3 R k\right]$ variables when $k$ is odd, and $\left[48 R^{2} k^{3} \log 3 R k^{2}\right]$ variables when $k$ is even. These results have recently been improved upon by Schmidt [22] and Low, Pitman and Wolff [21].

When $R=3$ the "Artin question" is whether $3 k^{2}+1$ variables are sufficient to ensure non-trivial $p$-adic solutions for every prime $p$. In the case $k=2$ this was proved by Ellison [19]. When $k=3$ Stevenson [23] showed that, except possibly for $p=3$ or $7, n \geq 28$ variables are sufficient. More recently Atkinson [1] has shown that 25 variables are sufficient to ensure non trivial $p$-adic solutions of three additive cubics in every $p$-adic field, except possibly $p=3$ or 7 .

We are indebted to the referee for many useful comments which have improved the exposition of our results.

\section{Preliminaries to Theorem 1}

We begin by recalling a normalisation procedure introduced by Davenport and Lewis $[12,14,15]$. With a pair (9) of additive forms $f, g$ we associate the parameter

$$
\theta=\theta(f, g)=\prod_{i \neq j}\left(a_{i} b_{j}-a_{j} b_{i}\right)
$$

For a given pair of forms with $\theta(f, g) \neq 0$ and a fixed prime $p$, there is a related $p$-normalized pair of forms $\left(f^{*}, g^{*}\right)$. Further the equations $f=g=0$ 
have a non-trivial $p$-adic solution if and only if the equations $f^{*}=g^{*}=0$ do. Also, by the $p$-adic compactness argument in Davenport and Lewis [14, Section 5], it is sufficient to prove Theorem 1 with the additional assumption that $\theta \neq 0$. We may now suppose that the forms $f, g$ are $p$-normalized, with $\theta \neq 0$, and use the following property which is essentially Lemma 2 of Davenport and Lewis [12].

LemMa 1. Let $f$ and $g$ be a p-normalized pair of forms. Then we may write

$$
\begin{aligned}
& f=f_{0}+p f_{1}, \\
& g=g_{0}+p g_{1} .
\end{aligned}
$$

Here $f_{0}, g_{0}$ are forms in $m \geq n / k$ variables, each of which occurs in one at least of $f_{0}, g_{0}$ with a coefficient not divisible by $p$. Further, if $q$ denotes the minimum number of variables occuring explicitly in any form $\lambda f_{0}+\mu g_{0}(\lambda, \mu$ not both divisible by $p$ ) with a coefficient not divisible by $p$, then $q \geq n / 2 k$.

Our next lemma is a version of Hensel's Lemma; it is Lemma 7 of Davenport and Lewis [14].

LEMMA 2. If $p \nmid k$ and the congruences

$$
\begin{array}{ll}
f_{0}=a_{1} x_{1}^{k}+\cdots+a_{m} x_{m}^{k} \equiv 0 & \bmod p, \\
g_{0}=b_{1} x_{1}^{k}+\cdots+b_{m} x_{m}^{k} \equiv 0 & \bmod p
\end{array}
$$

have a solution $\xi=\left(\xi_{1}, \ldots, \xi_{m}\right)$ for which the matrix

$$
\left(\begin{array}{c}
a_{1} \xi_{1} \cdots a_{m} \xi_{m} \\
b_{1} \xi_{1} \cdots b_{m} \xi_{m}
\end{array}\right)
$$

has rank $2(\bmod p)$ then the equations $f_{0}=g_{0}=0$ have a non-trivial solution in p-adic integers.

In the proof of Theorem 1 we have $p>k^{6}$ so $p \nmid k$. It is therefore sufficient to show that the congruences $(12)$ have a solution of rank $2(\bmod p)$. We may also suppose that $p \equiv 1 \bmod k$, see Lemma 3 of Davenport and Lewis [9]; similarly we may suppose that $p \equiv 1 \bmod 5$ for Theorem 2 .

Since $n>4 k$, Lemma 1 gives the bounds $m \geq 5, q \geq 3$. We partition the variables $x_{1}, \ldots, x_{m}$ into blocks such that in each block the ratios $a_{i} / b_{i}$ are equal $(\bmod p)$. Let $r$ be the length of the longest block of common ratios $a_{i} / b_{i}$. We note that replacing $f_{0}, g_{0}$ by suitable linear combinations we may take $a_{i} / b_{i}=$ " $1 / 0$ " for these $r$ variables. Further, let $t$ be the length of the second longest block of common ratios. We may take the ratios in this block to be " $0 / 1 "$. 
We assert that if $t \geq 3$ then the congruences (12) have a common solution of rank 2. This follows from our remarks on the single congruence (3) since the congruences (12) contain two disjoint congruences in 3 variables. Now we assume that $t \leq 2$ and reduce $m$ from its initial value to 5 by discarding variables from the longest block of common ratios. We end up with a pair of congruences (12) satisfying

$$
m=5, \quad q \geq 3 \text { and } r \leq 2
$$

since $r=m-q$.

\section{Exponential sums}

Since $r \leq 2$ we may renumber the variables in (12) so that $\left\{a_{1} / b_{1}, a_{2} / b_{2}\right\}$ and $\left\{a_{3} / b_{3}, a_{4} / b_{4}, a_{5} / b_{5}\right\}$ are sets of unequal ratios $\bmod p$. We count the number $N$ of solutions of the congruences (12) using exponential sums:

$$
N=p^{-2} \sum_{u_{1}, u_{2}} \sum_{\bmod p} T\left(\Lambda_{1}\right) \cdots T\left(\Lambda_{5}\right)
$$

where

$$
\begin{gathered}
\Lambda_{j}=u_{1} a_{j}+u_{2} b_{j}, \quad j=1, \ldots, 5, \\
T(\Lambda)=\sum_{x \bmod p} e\left(\Lambda x^{5} / p\right),
\end{gathered}
$$

and $e(\theta)=\exp (2 \pi i \theta)$.

Separating out the term $u_{1}=u_{2}=0$ in (15) we find that

$$
\begin{aligned}
N-p^{3} & =p^{-2} \sum^{\prime} T\left(\Lambda_{1}\right) \cdots T\left(\Lambda_{5}\right) \\
& =p^{-2}\left(\sum_{1}+\sum_{2}\right)
\end{aligned}
$$

where $\Sigma^{\prime}$ denotes the omission of the term $u_{1}=u_{2}=0, \Sigma_{1}$ is the sum over those terms for which no $\Lambda_{i} \equiv 0$ and $\sum_{2}$ is the sum over those terms $\left(u_{1}, u_{2}\right) \neq(0,0)$ for which some $\Lambda_{i} \equiv 0$.

Now

$$
\left|\sum_{1}\right|^{2} \leq \sum_{1}\left|T\left(\Lambda_{1}\right) T\left(\Lambda_{2}\right)\right|^{2} \cdot \sum_{1}\left|T\left(\Lambda_{3}\right) T\left(\Lambda_{4}\right) T\left(\Lambda_{5}\right)\right|^{2}
$$

We put

$$
S_{r}=\sum_{u=1}^{p-1}|T(u)|^{r} .
$$


Since $\Lambda_{1}, \Lambda_{2}$ are independent linear forms the mapping $\left(\Lambda_{1}, \Lambda_{2}\right) \rightarrow\left(u_{1}, u_{2}\right)$ is a bijection and therefore

$$
\sum_{1}\left|T\left(\Lambda_{1}\right) T\left(\Lambda_{2}\right)\right|^{2} \leq \sum_{\Lambda_{1} \Lambda_{2} \neq 0}\left|T\left(\Lambda_{1}\right) T\left(\Lambda_{2}\right)\right|^{2}=\sum_{1}\left|T\left(u_{1}\right) T\left(u_{2}\right)\right|^{2}=S_{2}^{2}
$$

Similarly, using Hölder's inequality, we have

$$
\begin{aligned}
\sum_{1}\left|T\left(\Lambda_{3}\right) T\left(\Lambda_{4}\right) T\left(\Lambda_{5}\right)\right|^{2} & \leq \max _{\Lambda_{i} \neq \Lambda_{j}} \sum_{1}\left|T\left(\Lambda_{i}\right) T^{2}\left(\Lambda_{j}\right)\right|^{2} \\
& =\sum_{u_{1}}\left|T\left(u_{1}\right)\right|^{2} \cdot \sum_{u_{2}}\left|T\left(u_{2}\right)\right|^{4}=S_{2} S_{4} .
\end{aligned}
$$

Thus

$$
\left|\sum_{1}\right| \leq S_{2}^{3 / 2} S_{4}^{1 / 2}
$$

In order to estimate $\sum_{2}$ suppose first that the ratio $a_{5} / b_{5} \bmod p$ occurs only once amongst the $a_{i} / b_{i}$. Then the contribution of the points $\left(u_{1}, u_{2}\right)$ with $\Lambda_{5} \equiv 0$ to $\sum_{2}$ is at most

$$
\begin{aligned}
p \sum_{\Lambda_{5} \equiv 0}\left|T\left(\Lambda_{1}\right) \cdots T\left(\Lambda_{4}\right)\right| & \leq p \max _{i \neq 5} \sum_{\Lambda_{5} \equiv 0}\left|T\left(\Lambda_{i}\right)\right|^{4} \\
& =p \sum_{u=1}^{p-1}|T(u)|^{4}=p S_{4},
\end{aligned}
$$

since the mapping $\left(\Lambda_{i}, \Lambda_{5}\right) \rightarrow\left(u_{1}, u_{2}\right)$ is a bijection. If the ratio $a_{5} / b_{5}$ occurs twice amongst the $a_{i} / b_{i}$ a similar argument shows that the contribution is at most $p^{2} S_{3}$. Thus

$$
\left|\sum_{2}\right| \leq \max \left(5 p S_{4}, 3 p S_{4}+p^{2} S_{3}, p S_{4}+2 p^{2} S_{3}\right) .
$$

Now (see Dodson [17, Lemma 2.5.1]),

$$
S_{2}=(k-1) p(p-1)
$$

and (see Davenport [10, Lemma 12])

$$
|T(u)| \leq(k-1) \sqrt{p}, \quad u \not \equiv 0 \bmod p
$$

so that

$$
\left|S_{3}\right|<(k-1)^{2} p^{5 / 2}
$$

and

$$
\left|S_{4}\right|<(k-1)^{3} p^{3}
$$

Hence

$$
\begin{aligned}
p^{-2}\left|\sum_{1}+\sum_{2}\right| & <p^{-2}\left\{(k-1)^{3} p^{9 / 2}+2(k-1)^{2} p^{9 / 2}+(k-1)^{3} p^{4}\right\} \\
& <k^{3} p^{5 / 2}
\end{aligned}
$$

since $p>k^{6}$. 
Any solution of rank 1 occurs in a pair of linearly dependent columns and since $r \leq 2$ there are at most 2 such pairs of columns, each pair giving $5(p-1)$ solutions. Further there is one solution of rank 0 and so at most $10 p-9$ solutions of rank $<2$. Thus we obtain the required solution of rank 2 provided that $p^{3}-k^{3} p^{5 / 2} \geq 10 p$.

This is equivalent to

$$
h(p, k)=p^{2}-k^{3} p^{3 / 2}-10 \geq 0,
$$

and, for fixed $k, h(p, k)$ is an increasing function of $p$ so it is enough to verify (32) when $p=k^{6}+1$ :

$$
k^{12}+2 k^{6}-9-k^{12}\left(1+k^{-6}\right)^{3 / 2} \geq 0
$$

or

$$
\left(1+2 k^{-6}-9 k^{-12}\right)^{2} \geq\left(1+k^{-6}\right)^{3} .
$$

Writing $y$ for $k^{6}$, we obtain $H(y)=y^{3}-17 y^{2}-37 y+81 \geq 0$. Now $H^{\prime} \geq 0$ for $y \geq 37 / 3$ and the inequality is easily verified for $y \geq 2^{6}=64$, which completes the proof of Theorem 1 .

\section{Preliminary remarks for Theorem 2}

After Theorem 1, we only need to consider those primes $p<5^{6}=15625$. The quintic residues mod $p$ form a cyclic subgroup of the non-zero residue classes, and the value of the exponential sum $T(u)$ depends only on the coset in which $u$ lies. For each prime $p \equiv 1 \bmod 5$ with $p \leq 15625$ we find the least quintic non-residue $q \bmod p$, using a computer. Then $S=\left\{1, q, q^{2}, q^{3}, q^{4}\right\}$ is a set of representatives from the 5 cosets. Using double precision Fortran we calculate the absolute values of the exponential sums

$$
T_{i}=\left|\sum_{x \bmod p} e\left(q^{i-1} x^{5} / p\right)\right|, \quad i=1, \ldots, 5,
$$

and these values are checked using the identity

$$
\sum_{i=1}^{5} T_{i}^{2}=20 p
$$

As $u$ runs through $1,2, \ldots, p-1$ it falls into each coset exactly $(p-1) / 5$ times and so

$$
S_{r}=\left(\frac{p-1}{5}\right) \sum_{i=1}^{5} T_{i}^{r}
$$


In this way we calculate $S_{2}(=4 p(p-1)), S_{3}$ and $S_{4}$ exactly, and compute the bound

$$
B=S_{2}^{3 / 4} S_{4}^{1 / 2}+\max \left(5 p S_{4}, 3 p S_{4}+p^{2} S_{3}, p S_{4}+2 p^{2} S_{3}\right)
$$

for $\sum_{1}+\sum_{2}$. Then, checking the primes up to 15625 we obtain

$$
p^{3}-p^{-2} B \geq 10 p \text { for } 6800<p \leq 15625
$$

which leads to the required solution of rank 2 .

We now take $p \equiv 1 \bmod 5$ to be a fixed prime in the range

$$
11<p \leq 6800 \text {. }
$$

We find the least quintic non-residue $q \bmod p$ and put

$$
S=\left\{1, q, \ldots, q^{4}\right\}
$$

Lemma 3. Let $p \equiv 1 \bmod 5, p>11$. If $a b c \not \equiv 0 \bmod p$ then

$$
a x^{5}+b y^{5}+c z^{5} \equiv d \quad \bmod p
$$

has a solution, which is non-trivial if $d \equiv 0 \bmod p$.

Proof. For $d \not \equiv 0 \bmod p$ this follows from Theorem 3 of Chowla, Mann and Straus [5]. Now $d \equiv 0 \bmod p$ and for $p>625$ the result follows from Theorem 1 of I Chowla [3] (or Lemma 2.4.1 of Dodson [17]).

For $11<p \leq 625$, using substitutions $x \rightarrow \alpha x$, we may assume that $a, b, c \in S$. This result is obvious unless $a, b, c$ are unequal and we may suppose that

$$
1=a<b<c .
$$

Thus for each prime $p$ there are only 6 cases to consider and the result is easily verified by computer.

\section{Proof of Theorem 2(a)}

The normalization process described in Section 2 results in a pair of forms with $m=5, q \leq 3$ and $r \leq 2$, which we can write in the form

$$
\begin{aligned}
& f_{0}=x_{1}^{5}+a_{2} x_{2}^{5}+\cdots+a_{4} x_{4}^{5} \equiv 0 \bmod p, \\
& g_{0}=00 \bmod p
\end{aligned}
$$

where possibly $a_{4} \equiv 0 \bmod p$ but $a_{3} \not \equiv 0 \bmod p$, and $a_{2}, b_{3}, b_{4} \in S$. In this section we consider the case $r=2$. 
LeMMA 4. Let $p \equiv 1 \bmod 5, p \geq 101$. If $a b c \not \equiv 0 \bmod p$ then the congruence

$$
a x^{5}+b y^{5}+c z^{5} \equiv d \quad \bmod p
$$

has a solution with $x y z \not \equiv 0 \bmod p$.

Proof. We count the number $N_{1}$ of solutions of (43) using exponential sums:

$$
\left|N_{1}-p^{2}\right| \leq p^{-1} S_{3} \leq 16(p-1) \sqrt{p}
$$

using (27) and (28).

When $x \equiv 0$ the congruence (43) becomes

$$
b y^{5}+c z^{5} \equiv d \quad \bmod p .
$$

For any given value $y$ there are at most 5 solutions for $z$, so the number of solutions of (43) with $x y z \equiv 0 \bmod p$ is at most $15 p$. We have

$$
N_{1} \geq p^{2}-16 p^{3 / 2}>15 p
$$

for $p \geq 291$.

For $101 \leq p<291$ we take $a, b, c \in S$ with

$$
1=a \leq b \leq c
$$

(after substitutions $x \rightarrow \alpha x$ ). The result is now easily verified by computer.

Lemma 5. Let $p \equiv 1 \bmod 5, p \geq 101$. If $r=2$ then the congruences (42) have a solution of rank $2 \bmod p$.

Proof. We begin by solving

$$
b_{3} x_{3}^{5}+b_{4} x_{4}^{5}+x_{5}^{5} \equiv 0 \bmod p
$$

with $x_{3} x_{4} x_{5} \not \equiv 0 \bmod p$. This solution involves 2 linearly independent columns of coefficients.

Let

$$
A=a_{3} x_{3}^{5}+a_{4} x_{4}^{5}
$$

If $A \equiv 0$ we take $x_{1}=x_{2}=0$ to give the required solution. Otherwise we multiply $x_{3}, x_{4}, x_{5}$ by $\xi$ and solve

$$
x_{1}^{5}+a_{2} x_{2}^{5}+A \xi^{5} \equiv 0 \bmod p
$$

with $x_{1} x_{2} \xi \not \equiv 0 \bmod p$ to give the required solution.

We now take $t$ to be the length of the second longest block of common ratios $a_{i} / b_{i} \bmod p$. 
LEMMA 6. Let $p \equiv 1 \bmod 5, p>11$. If $r=2, t=1$ and $a_{2}$ is a quintic nonresidue $\bmod p$ then the congruences (42) have a solution of rank $2 \bmod p$.

Proof. This is a repetition of Lemma 5 except that the solution of (48) is non-trivial, but still involves two linearly independent columns, and the solution of $(50)$ has $\xi \not \equiv 0$ since $a_{2}$ is a quintic non-residue.

We are now left with the cases

$$
p=31,41,61 \text { or } 71 \text {; }
$$

either $r=2, t=2$, and then

$$
\begin{array}{ll}
f_{0} & =x_{1}^{5}+a_{2} x_{2}^{5}+a_{3} x_{3}^{5}, \\
g_{0} & =b_{3} x_{3}^{5}+b_{4} x_{4}^{5}+x_{5}^{5}
\end{array}
$$

where $a_{2}, b_{3}, b_{4} \in S, a_{3} \not \equiv 0 \bmod p$;

or $r=2, t=1, a_{2}=1$, and then

$$
\begin{array}{ll}
f_{0}=x_{1}^{5}+x_{2}^{5}+a_{3} x_{3}^{5}+a_{4} x_{4}^{5} \\
g_{0}=\quad b_{3} x_{3}^{5}+b_{4} x_{4}^{5}+x_{5}^{5},
\end{array}
$$

where $b_{3}, b_{4} \in S, a_{3} a_{4} \not \equiv 0 \bmod p$.

For a fixed prime $p$ there 25 forms $g_{0}$ to consider. For each $g_{0}$ we begin by forming a list of all solutions of $g_{0} \equiv 0 \bmod p$. We then run through $5(p-1)$ forms $f_{0}$ of the first type (52) and $(p-1)^{2}$ forms $f_{0}$ of the second type (54). The computer then runs through the list of solutions of $g_{0} \equiv 0 \bmod p$ until it finds one which is also a solution of $f_{0} \equiv 0 \bmod p$ and which has rank 2 . In this way a computer search revealed the counterexample listed in Section 8.

\section{Theorem 2(a): the case $r=1$}

In this case any non-trivial solution has rank $2 \bmod p$. We begin by writing the congruences as

$$
\begin{aligned}
& f_{0}=x_{1}^{5}+a_{2} x_{2}^{5}+\cdots+a_{5} x_{5}^{5} \equiv 0 \bmod p, \\
& g_{0}=\quad b_{2} x_{2}^{5}+\cdots+b_{5} x_{5}^{5} \equiv 0 \quad \bmod p,
\end{aligned}
$$

where $b_{2}, \ldots, b_{s} \in S$.

Suppose first that $b_{2}, \ldots, b_{5}$ consist of two pairs of equal values, say $b_{2}=b_{3}$ and $b_{4}=b_{5}$. We take $x_{2}=-x_{3}=u, x_{4}=-x_{5}=v$ and the non-trivial solution of

$$
x_{1}^{5}+\left(a_{2}-a_{3}\right) u^{5}+\left(a_{4}-a_{5}\right) v^{5} \equiv 0 \bmod p
$$


gives the required solution of rank 2. (The coefficients are non-zero since $r=1$.) Now we may assume that for any form $g^{*}$ in the pencil generated by $f_{0}, g_{0}$ and having one zero coefficient, the 4 non-zero coefficients do not all lie in the same coset.

We count the number $N_{2}$ of solutions of (56) using exponential sums. Since the ratios $a_{i} / b_{i}$ are distinct $\bmod p$ we have, as in Section 3,

$$
\begin{aligned}
N_{2}-p^{3} & =p^{-2} \sum_{u_{1}, u_{2}}^{\prime} T\left(\Lambda_{1}\right) \cdots T\left(\Lambda_{5}\right) \\
& =p^{-2}\left(\sum_{1}+\sum_{2}\right) .
\end{aligned}
$$

Here $\sum_{1}$ is the contribution coming from those points $\left(u_{1}, u_{2}\right)$ for which no $\Lambda_{i} \equiv 0 \bmod p$. Now

$$
\left|\sum_{1}\right|^{2} \leq \sum_{1}\left|T\left(\Lambda_{1}\right) T\left(\Lambda_{2}\right)\right|^{2} \cdot \sum_{1}\left|T\left(\Lambda_{3}\right) T\left(\Lambda_{4}\right) T\left(\Lambda_{5}\right)\right|^{2} .
$$

Since $\Lambda_{1}$ and $\Lambda_{2}$ are linearly independent the first sum on the right factorizes to give $S_{2}^{2}$. The second sum is majorized by

$$
\max _{i \neq j} \sum_{1}\left|T\left(\Lambda_{i}\right) T\left(\Lambda_{j}\right)\right|^{3}=S_{3}^{2}
$$

Hence

$$
\left|\sum_{1}\right| \leq S_{2} S_{3}
$$

The term $\sum_{2}$ in (58) is the contribution coming from those points $\left(u_{1}, u_{2}\right)$ for which some $\Lambda_{i} \equiv 0 \bmod p$.

LEMMA 7. We have

$$
\left|\sum_{2}\right| \leq 5 p S_{4}
$$

Proof. Since $\Lambda_{1}=u_{1}$ the contribution to $\sum_{2}$ coming from the terms with $\Lambda_{1} \equiv 0 \bmod p$ is at most

$$
\left|p \sum_{u=1}^{p-1} T\left(b_{2} u\right) \cdots T\left(b_{5} u\right)\right| \leq p \prod_{i=2}^{5}\left\{\sum_{u=1}^{p-1}\left|T\left(b_{i} u\right)\right|^{4}\right\}^{1 / 4} .
$$

As $u$ runs through $1,2, \ldots, p-1$ so does $b_{i} u$. Thus each of these sums

$$
\sum_{u=1}^{p-1}\left|T\left(b_{i} u\right)\right|^{4}=\sum_{u=1}^{p-1}|T(u)|^{4}=S_{4}
$$

so this contribution to $\sum_{2}$ is majorized by

$$
p S_{4} \text {. }
$$


We assert that the same bound applies to the contribution arising from the points $\left(u_{1}, u_{2}\right)$ with $\Lambda_{j} \equiv 0 \bmod p$ for each $j=2, \ldots, 5$. If $\Lambda_{j} \equiv 0 \bmod p$ then, interpreting $b_{j}^{-1} \bmod p, u_{2} \equiv-a_{j} u_{1} / b_{j} \bmod p$ and so for $i \neq j$

$$
\begin{aligned}
\Lambda_{i} & \equiv u_{1}\left(a_{i} b_{j}-a_{j} b_{i}\right) / b_{j} \quad \bmod p \\
& =c_{i} u_{i}
\end{aligned}
$$

say. Thus the contribution of these terms is

$$
p \sum_{u=1}^{p-1} \prod_{i \neq j} T\left(c_{i} u_{i}\right)
$$

Now we can replace $f_{0}, g_{0}$ in (56) by any 2 independent forms in the pencil, for example by $f^{*}=f_{0}$ and

$$
g^{*} \equiv\left(b_{j} f_{0}-a_{j} g_{0}\right) / b_{j} \quad \bmod p .
$$

The coefficients $c_{i}$ are just the coefficients of $g^{*}$ and therefore (67) is also bounded by (65), which gives the lemma.

The estimates (58), (61) and (63) give

$$
N_{2} \geq p^{3}-S_{2} S_{3}-5 p S_{4} \text {. }
$$

For $11<p<6800$ we calculate the bound on the right of (69) and find that $N_{2}>1$ (implying a non-trivial solution, which will have rank 2) for $p>3061$.

\section{Proof of Theorem 2(b)}

Now $n \geq 26$ so

$$
m \geq 6, \quad q \geq 3 .
$$

Discarding excess variables we may take $m=6$ and still have $q \geq 3$, so $r \leq 3$. We suppose first that $r=1$, and therefore any non-trivial solution of the congruences (14) has rank 2 . We begin by rewriting the congruences in the form

$$
\begin{aligned}
f_{0}=x_{1}^{5}+a_{2} x_{2}^{5}+\cdots+a_{6} x_{6}^{5} \equiv 0 & \bmod p, \\
g_{0}=\quad b_{2} x_{2}^{5}+\cdots+b_{6} x_{6}^{5} \equiv 0 & \bmod p
\end{aligned}
$$

where $b_{2}, \ldots, b_{6} \in S$.

Suppose first that some value is repeated amongst $b_{2}, \ldots, b_{6}$; then we may take $b_{2}=b_{6}=1$. Replacing $f_{0}$ by $b_{6} f_{0}-a_{6} g_{0}$ we may also take $a_{6}=0$. Consider any non-trivial solution of the congruence

$$
b_{3} x_{3}^{5}+b_{4} x_{4}^{5}+b_{5} x_{5}^{5} \equiv 0 \quad \bmod p .
$$


If

$$
A=a_{3} x_{3}^{5}+a_{4} x_{4}^{5}+a_{5} x_{5}^{5} \equiv 0 \quad \bmod p
$$

then we have the required solution. Otherwise we multiply $x_{3}, x_{4}, x_{5}$ by $\xi$, take $x_{2}=-x_{6}=u$ and solve

$$
x_{1}^{5}+a_{2}^{5} u+A \xi^{5} \equiv 0 \quad \bmod p
$$

to give the required solution.

We may now suppose that $b_{2}, \ldots, b_{6}$ lie one in each of the distinct cosets. Similarly, for any form $g^{*}$ in the pencil generated by $f_{0}$ and $g_{0}$ which has one zero coefficient, the other 5 coefficients must lie one in each coset. Counting the number $N_{2}$ of solutions of (71) using exponential sums we have

$$
\begin{aligned}
N_{2}-p^{4} & =p^{-2} \sum^{\prime} T\left(\Lambda_{1}\right) \cdots T\left(\Lambda_{6}\right) \\
& =p^{-2}\left(\sum_{1}+\sum_{2}\right),
\end{aligned}
$$

where $\sum_{1}$ is the sum over those $\left(u_{1}, u_{2}\right)$ for which no $\Lambda_{i} \equiv 0 \bmod p$ and $\sum_{2}$ is the sum over those $\left(u_{1}, u_{2}\right)$ for which some $\Lambda_{i} \equiv 0 \bmod p$.

Since $r=1$ we have

$$
\left|\sum_{1}\right| \leq S_{3}^{2}
$$

The contribution to $\sum_{2}$ coming from the points $\left(u_{1}, u_{2}\right)$ with $\Lambda_{1}=u_{1} \equiv 0$ $\bmod p$ is at most

$$
\left|p \sum_{u=1}^{p-1} T\left(b_{2} u\right) \cdots T\left(b_{6} u\right)\right| \leq p(p-1) T_{1} \cdots T_{5} .
$$

As in Section 6 the same estimate holds on each line $\Lambda_{j} \equiv 0 \bmod p$ so

$$
\left|\sum_{2}\right| \leq 6 p(p-1) T_{1} \cdots T_{5} \text {. }
$$

For $131<p \leq 3061$ we find that

$$
p^{4}-S_{3}^{3}-6 p(p-1) T_{1} \cdots T_{5}>1
$$

so $N_{2}>1$, and we have the required solution.

Each of the remaining primes has $q=2$ so we may take

$$
g_{0}=x_{2}^{5}+2 x_{3}^{5}+4 x_{4}^{5}+8 x_{5}^{5}+16 x_{6}^{5} \text {, }
$$

and we begin by forming a list of non-trivial solutions of $g_{0} \equiv 0 \bmod p$. We may take $f_{0}$ to be form with $a_{6}=0, a_{1}=1$ and the other coefficients lying one in each coset. If $A, B, C, D$ are representatives of the cosets then $a_{2}, \ldots, a_{5}$ is of type $A, B, C, D$ in some order, giving 24 different cases for $f_{0}$. For each of these cases there are $((p-1) / 5)^{4}$ individual forms $f_{0}$ to 
consider. The computer runs through each of these and then runs down the list of non-trivial solutions of $g_{0} \equiv 0 \bmod p$ until it finds a common solution (since $r=1$ this solution must have rank 2).

If $r=3$ the congruences become

$$
\begin{aligned}
& f_{0}=a_{1} x_{1}^{5}+a_{2} x_{2}^{5}+a_{3} x_{3}^{5}+\cdots+a_{6} x_{6}^{5} \quad \equiv 0 \quad \bmod p, \\
& g_{0}=\quad b_{4} x_{4}^{5}+\cdots+b_{6} x_{6}^{5} \equiv 0 \quad \bmod p
\end{aligned}
$$

where $a_{1}, a_{2}, a_{3}, b_{4}, b_{5}, b_{6} \not \equiv 0 \bmod p$. We solve $g_{0} \equiv 0$ with $x_{4}, x_{5}, x_{6}$ not all zero, and then solve $f_{0} \equiv 0$ with $x_{1}, x_{2}, x_{3}$ not all zero. This solution has rank 2.

Now we are left with the case $r=2$. We discard one of $x_{3}, \ldots, x_{6}$ to reduce the problem to the case

$$
m=5, \quad r=2, \quad q=3
$$

already contained in Section 5 . The results of Section 5 provide the required solution when $p \geq 101$ and we are now left with the primes $31,41,61$ and 71.

We repeat the argument used at the end of Section 5; either

(i) $r=t=2$ and then

$$
\begin{aligned}
& f_{0}=x_{1}^{5}+a_{2} x_{2}^{5}+a_{3} x_{3}^{5}+a_{4} x_{4}^{5}, \\
& g_{0}=\quad b_{3} x_{3}^{5}+b_{4} x_{4}^{5}+b_{5} x_{5}^{5}+x_{6}^{5}
\end{aligned}
$$

where $a_{2}, b_{3}, b_{4}, b_{5} \in S_{1}, a_{3} a_{4} \not \equiv 0 \bmod p$; or

(ii) $r=2, t=1, a_{2}=1$ and then

$$
\begin{array}{ll}
f_{0}=x_{1}^{5}+x_{2}^{5}+a_{3} x_{3}^{5}+a_{4} x_{4}^{5}+a_{5} x_{5}^{5}, \\
g_{0}=\quad b_{3} x_{3}^{5}+b_{4} x_{4}^{5}+b_{5} x_{5}^{5}+x_{6}^{5}
\end{array}
$$

where $b_{3}, b_{4}, b_{5} \in S, a_{3} a_{4} a_{5} \not \equiv 0 \bmod p$.

For a fixed prime $p$ there are 125 forms $g_{0}$ to consider. For each $g_{0}$ we begin by forming a list of solutions of $g_{0} \equiv 0 \bmod p$. We then run through $5(p-1)^{2}$ forms of the first type (84) and $(p-1)^{3}$ forms of the second type (86). The computer then runs through the list of solutions of $g_{0} \equiv 0 \bmod p$ until it finds one which is also a solution of $f_{0} \equiv 0 \bmod p$ and which has rank $2 \bmod p$. In this way a compter (the IBM 3083 at Sheffield University) completed the proof of Theorem 2.

\section{Some counterexamples}

The computer search described in Sections 5 and 6 produced the following counterexamples with $m=5$ : 
(i) $p=31$,

$$
\begin{aligned}
& f_{0}=x_{1}^{5}+x_{2}^{5}+x_{3}^{5}+3 x_{4}^{5} \\
& g_{0}=2 x_{2}^{5}+4 x_{3}^{5}+2 x_{4}^{5}+x_{5}^{5}
\end{aligned}
$$

(ii) $p=41$,

$$
\begin{aligned}
& f_{0}=x_{1}^{5}+x_{2}^{5}+x_{3}^{5}+2 x_{4}^{5}, \\
& g_{0}=2 x_{2}^{5}+4 x_{3}^{5}+22 x_{4}^{5}+x_{5}^{5}
\end{aligned}
$$

(iii) $p=61$, when there are only singular solutions,

$$
\begin{array}{ll}
f_{0}=x_{1}^{5}+x_{2}^{5}+4 x_{3}^{5} \\
g_{0}= & 4 x_{3}^{5}+2 x_{4}^{5}+x_{5}^{5} .
\end{array}
$$

It is well known that the $p$-adic fields with $p=5,11$ are exceptional for quintic equations. However the counterexamples above are of a different character. The problem here is simply that the prime $p$ is too small rather than it being of any generic type $(p=k$ or $2 k+1)$.

\section{References}

[1] O. D. Atkinson (PhD Dissertation, University of Sheffield, 1989).

[2] R. C. Baker and J. Brüden, 'On pairs of additive cubic equations', J. Reine Angew. Math. 391 (1988), 157-180.

[3] Z. I. Borevich and I. R. Shafarevich, Number theory (Academic Press, New York, 1966).

[4] I. Chowla, 'On the number of solutions of some congruences in two variables', Proc. Nat. Acad. Sci. India Ser. A 5 (1937), 40-44.

[5] S. Chowla, H. B. Mann and E. G. Straus, 'Some applications of the Cauchy-Davenport theorem', Norske Vid. Selsk. Forh. 32 (1959), 74-80.

[6] R. J. Cook, 'Pairs of additive equations', Michigan Math. J. 19 (1972), 325-331.

[7] R. J. Cook, 'Pairs of additive congruences: cubic congruences', Mathematika 32 (1985), 286-300.

[8] R. J. Cook, 'Pairs of additive congruences: quintic congruences', Indian J. Pure Appl. Math. 17 (1986), 786-799.

[9] R. J. Cook, 'Computations for additive Diophantine equations: quintic congruences II', Computers in Mathematical Research, edited by N. M. Stephens and M. P. Thorne, pp. 93-117 (Clarendon Press, Oxford, 1988).

[10] H. Davenport, Analytic methods for Diophantine equations and Diophantine inequalities (Campus Publishers, Ann Arbor, 1963).

[11] H. Davenport and D. J. Lewis, 'Homogeneous additive equations', Proc. Roy. Soc. London Ser A 274 (1963), 443-460.

[12] H. Davenport and D. J. Lewis, 'Cubic equations of additive type', Philos. Trans. Roy. Soc. London Ser. A 261 (1966), 97-136.

[13] H. Davenport and D. J. Lewis, 'Notes on congruences III', Quart. J. Math. Oxford Ser (2), 17 (1966), 339-344. 
[14] H. Davenport and D. J. Lewis, 'Two additive equations', Proc. Sympos. Pure Math. 12 (1967), 74-98.

[15] H. Davenport and D. J. Lewis, 'Simultaneous equations of additive type', Philos. Trans. Roy. Soc. London Ser. A 264 (1969), 557-595.

[16] V. B. Demyanov, 'Pairs of quadratic forms over a complete field with discrete norm with finite residue class field', Izv. Akad. Nauk SSSR 20 (1956), 307-324.

[17] M. M. Dodson, 'Homogeneous additive congruences', Philos. Trans. Roy. Soc. London Ser A 261 (1966), 163-210.

[18] F. Ellison, 'Three diagonal quadratic forms', Acta Arith. 23 (1973), 137-151.

[19] J. F. Gray, Diagonal forms of prime degree (PhD thesis, University of Notre Dame, 1958).

[20] D. J. Lewis, 'Cubic congruences', Michigan Math. J. 4 (1957), 85-95.

[21] L. Low, J. Pitman and A. Wolff, 'Simultaneous diagonal congruences', J. Number Theory 29 (1988), 31-59.

[22] W. M. Schmidt, 'The solubility of certain p-adic equations', J. Number Theory 19 (1984), 63-80.

[23] E. Stevenson, 'The Artin conjecture for three diagonal cubic forms', J. Number Theory 14 (1982), 374-390.

[24] R. C. Vaughan, 'On pairs of additive cubic equations', Proc. London Math. Soc. 34 (1977), 354-364.

[25] R. C. Vaughan, 'On Waring's problem for smaller exponents', Proc. London Math. Soc. 52 (1986), 445-463.

Department of Pure Mathematics

University of Sheffield

Sheffield S3 7RH

England 